\title{
Enhanced Stability of a Carbon Monoxide Monolayer Adsorbed on Platinum under Electrochemical Control probed by Sum-Frequency Generation Spectroscopy
}

\author{
Christophe Humbert, Bertrand Busson, Abderrahmane Tadjeddine*
}

Laboratoire de Chimie Physique, CNRS, Univ. Paris-Sud, Université Paris-Saclay, Bâtiment 201 Porte 2, 91405 Orsay, France

\begin{abstract}
We establish a new procedure to study the steady state adsorption of carbon monoxide (CO) at the surface of platinum under electrochemical control over a wide potential range in aqueous acidic solution. Using highly concentrated solutions of $\mathrm{CO}$ in perchloric acid medium enables the formation of a tightly packed and very stable $\mathrm{CO}$ monolayer at the surface of the metal, which allows cyclic voltammetry in the $[-1200 ;+1500 \mathrm{mV}]$ potential range (vs $\mathrm{Ag} / \mathrm{AgCl})$. We use Sum-Frequency Generation (SFG) spectroscopy to show the unusual stability of the monolayer: going towards negative potentials, hydrogen evolution is first blocked then strongly quenched; towards positive potentials, CO oxidation is prevented as a consequence of its dense packing as a "frozen" monolayer. Diluting CO concentration by a factor of ten is enough to recover most of the usual properties of the $\mathrm{CO} / \mathrm{Pt}(110)$ interface, still maintaining the high stability of the $\mathrm{CO}$ adlayer. Such a protocol can be applied to any problem for which CO poisoning mechanism has to be scrutinized or as a model of very stable electrochemical interfaces for fundamental studies.
\end{abstract}

\section{Introduction}

The CO on platinum system has been intensively studied as a model surface reaction in gas phase as well as in the electrochemical environment. One key issue is the poisoning effect of $\mathrm{CO}$, as an intermediate adsorbed species of organics oxidation. This phenomenon acts as a limiting factor for the performance of fuel cells ${ }^{1,2}$ based on alcohol reactants ${ }^{3}$ in acidic aqueous solutions. Whatever the environment where they occur (gas, liquid), the monitoring and fundamental understanding of these molecular reactions at the surface of the catalyst require the use of sensitive and selective analytical and spectroscopic tools. ${ }^{4-13}$

Nonlinear Sum-Frequency Generation spectroscopy $(\mathrm{SFG})^{14-22}$ is a powerful in situ probe of the surface coverage and adsorption geometry in a reactor ${ }^{23-28}$, as well as in a spectro-electrochemical cell. In the latter case, the electric potential applied across the surface controls the chemical reaction and the structure of the electrodeelectrolyte interface ${ }^{29}$. SFG is an absolute investigation tool since it does not require the measurement of a blank spectrum ${ }^{30}$. In addition, the interferences between the vibrationally resonant and non resonant contributions, specific to the technique, lead to additional local information, like the absolute molecular orientation ${ }^{31}$, the sign of the vibrational dynamic dipole moment ${ }^{25}$ or, as will be shown below, the determination of the potential of zero charge (PZC) of the system ${ }^{32,33}$. In a model alcohol fuel cell, $\mathrm{CO}$ poisoning hampers catalytic efficiency inside a specific potential range, which depends on the concentration of alcohol reactants and the $\mathrm{pH}$ composition of the electrolyte. In addition, the $\mathrm{CO}$ surface coverage $(\Theta)$ also depends on the potential because of the presence of complex reaction intermediates, which makes spectroscopic data interpretation less straightforward ${ }^{34}$. Understanding the mechanism and stability of $\mathrm{CO}$ poisoning requires a fine analysis of the $\mathrm{CO} / \mathrm{Pt}$ system as a function of platinum crystallinity and surface coverage over a wide potential range. It is common to perform electrochemical measurements in an aqueous solution (e.g. with perchloric acid $\mathrm{HClO}_{4}$ ), previously saturated with high purity grade gaseous $\mathrm{CO}$. In these conditions, only $\mathrm{CO}$ molecules interact with the electrode's surface provided that one works out of the electrolyte reduction and oxidation ranges. To explain a dependency of the vibrational spectra on $\Theta$, one has to take into account the dipoledipole interaction, whose delocalized character increases with $\Theta .{ }^{35}$ The situation is complicated at low CO coverage by the co-adsorption of water and hydrogen. Moreover, the vibrational frequencies of adsorbed species depend on the static electric field, via the Stark effect. ${ }^{34,36,37}$ It becomes even more complicated when both $\Theta$ and the interface electric field change simultaneously, which is often the case, especially at low CO coverage, where they both depend on the applied potential. Finally, the accessible potential range in an aqueous solution spans only about $0.8 \mathrm{~V}$, between hydrogen evolution at lower potentials and $\mathrm{CO}$ oxidation at higher ones ${ }^{38}$.

To overcome this limitation, we and other teams have worked in $\mathrm{CO}$ saturated nonaqueous (e.g. acetonitrile) oxygen-free solutions ${ }^{38,39,40}$, hence extending the working 
potential range from $0.8 \mathrm{~V}$ to about $2.7 \mathrm{~V}$. The Weaver group could consequently measure the variation of the Stark slope over a wide potential range as a function of the solvent and the nature of the counter ions ${ }^{38,39}$, explaining the observed values by the varying thickness of the effective double layer. In a previous work, ${ }^{40}$ we have investigated the effect of the electric field across the interface on the vibrational and electronic properties of a $\mathrm{CO}$ adlayer on a $\mathrm{Pt}(110)$ electrode (for which only atop $\mathrm{CO}$ exists, with a high coverage) in such a nonaqueous electrolyte, using a combination of IR and SFG in situ techniques. The dependence of the $\mathrm{CO}$ vibrational properties on both $\Theta$ and the static electric field allowed us to show that dipole-dipole coupling among $\mathrm{CO}$ molecules in the adlayer should be taken into account to explain the influence of the electric field, and that the observed evolution of the interfacial properties as a function of the potential were due to changes in the electronic and vibrational polarizabilities of the $\mathrm{CO}$ molecule.

In the present work, we show that it is possible to extend the potential range of a stable $\mathrm{CO} / \mathrm{Pt}(110)$ interface in an aqueous medium. To reach that goal, we take advantage of a very concentrated and stable CO-saturated acidic solution as the electrolyte in a thin electrolyte layer configuration. We investigate the peculiar properties of this interface by IR-Visible SFG spectroscopy, showing its originality in terms of Stark shift of the $\mathrm{CO}$ internal stretch and very high molecular packing, as well as quenching of both the hydrogen evolution and $\mathrm{CO}$ oxidation processes. A tenfold dilution of this mother solution leads to recover most of the usual electrochemical and vibrational properties of a $\mathrm{CO}$ on $\mathrm{Pt}(110)$ interface in contact with an acidic $\mathrm{CO}$ saturated solution.

\section{EXPERIMENTAL}

Preparation of the $\mathrm{CO}$ solution and Platinum electrode: In order to build our model catalyst interface, a CO-saturated solution is obtained by trapping pure $\mathrm{CO}$ gas (Messer, 99.997\%) in $\mathrm{HClO}_{4}$ aqueous solution (SigmaAldrich, o.1M) for at least 30 minutes ( 5 bubbles/second) in a sealed cleaned glass container. Using a sealed container allows to work with a solution in contact with a high pressure $\mathrm{CO}$ atmosphere, leading to the dissolution of more $\mathrm{CO}$ molecules into the liquid. The whole experimental process (saturation of the solution, dilution, filling of the electrochemical cell and recording of SFG spectra) was done without any contact with ambient atmosphere, in sealed containers. In particular, the electrochemical cell was kept full of solution with no contact with ambient air. In these conditions, the high $\mathrm{CO}$ concentration in the resulting solution remains stable for 48 hours. A second solution, obtained after dilution of the original concentration by a factor of ten in $\mathrm{HClO}_{4}$ (o.1M) buffer, will be referred to as the $\mathrm{CO}(\mathrm{C} / 10)$ solution. The $\mathrm{CO}$ concentration is however still higher than in reference 4o. A high purity grade platinum electrode, $\mathrm{Pt}(110)$ from Goodfellow ( $1 \mathrm{~cm}$ diameter for $1 \mathrm{~mm}$ thickness), is firstly mechanically polished until optical finishing with diamond paste. It is then dipped in a piranha bath for 10 minutes to remove the polishing residues from the surface and abundantly rinsed with Millipore water $(18 \mathrm{M} \Omega . \mathrm{cm})$. Afterwards, the electrode is flame-annealed (red-orange optical emission in a dark room) for 10 minutes at ambient air in order to favor the (110)-facetted reconstruction of the surface ${ }^{25}$ and activate it for the adsorption of a CO monolayer. After cooling for a couple of minutes in the same conditions, a drop of $\mathrm{CO}$-saturated or $\mathrm{CO}(\mathrm{C} / 10) \mathrm{HClO}_{4}$ solution protects the Pt surface prior to its immersion in the spectroelectrochemical cell. The cell is filled with the aforementioned $\mathrm{CO}$-saturated or $\mathrm{CO}(\mathrm{C} / 10) \mathrm{HClO}_{4}$ aqueous electrolytic solutions depending on the degree of $\mathrm{CO}$ saturation. The electrode is gently pressed against a calcium fluoride $\left(\mathrm{CaF}_{2}\right)$ prism closing the spectro-electrochemical cell and ensuring that the incident IR beam used in the SFG process reaches the probed surface with minimal absorption. The working potential is set to $+10 \mathrm{mV}$, then decreased to $-200 \mathrm{mV}$ vs. $\mathrm{Ag} / \mathrm{AgCl}$ reference electrode plunged into a saturated $\mathrm{KCl}$ solution $(3 \mathrm{M})$ to maintain a stable $\mathrm{CO}$ monolayer as a starting point. At this potential, hydrogen adsorption-desorption competing processes are expected to occur without denaturing the surface structure of the electrode. All the potentials in the following refer to this reference electrode. Current density curves are recorded as a function of the applied potential in the thin electrolyte configuration by a scanning potentiostat (EG\&G Instruments) in cyclic voltammetry conditions.

Sum-Frequency Generation spectroscopy: The spectro-electrochemical cell used for the experiment is detailed in a previous publication. ${ }^{41}$ The SFG photons are produced after mixing the infrared $(4.7-5.2 \mu \mathrm{m})$ and visible $(532 \mathrm{~nm})$ laser beams at the same point of the probed interface with incidence angles of $65^{\circ}$ and $55^{\circ}$ with respect to the surface normal, respectively. The experimental setup used is described elsewhere. ${ }^{42}$ It is based on a $\mathrm{Nd}: \mathrm{YVO}_{4}$ pulsed (6.8 ps) laser source at $1064 \mathrm{~nm}$ coupled to an acousto-optic modulator in order to temporally shape the laser beam $(62.5 \mathrm{MHz}$ micropulse repetition rate in a $2 \mu \mathrm{s}$ train with a $25 \mathrm{~Hz}$ macropulse repetition rate). After amplification, one part of the beam pumps the infrared optical parametric oscillator built around an $\mathrm{AgGaS}_{2}$ crystal (4.5-8 $\mu \mathrm{m}, 2 \mathrm{~cm}^{-1}$ bandwidth FWHM) while the other part is used to pump a visible optical parametric oscillator built around a BBO crystal $\left(420-720 \mathrm{~nm}, 6 \mathrm{~cm}^{-1}\right.$ bandwidth) after frequency up-conversion at $355 \mathrm{~nm}$ of the $1064 \mathrm{~nm}$ fundamental beam through SHG and THG stages in BBO and LBO crystals, respectively. All beams are p-polarized (SFG, Visible, Infrared) during the experiments. The SFG data are normalized by a reference SFG signal generated in the bulk of a $\mathrm{ZnS}$ crystal in order to compensate for atmospheric water absorption bands in the probed IR spectral range. A typical SFG spectrum, for which the infrared wavenumber is scanned between 1920 and $2130 \mathrm{~cm}^{-1}$, requires between 18 and 20 minutes to record. For a series of SFG spectra, the potential is scanned down from the starting point towards negative potentials, then up again until the end potential in the positive region.

\section{RESULTS AND DISCUSSION}


Cyclic voltammetry (Figure 1) allows to establish the potential range of interest for the $\mathrm{CO}$ adsorption on the metal between hydrogen evolution at low potential $(\mathrm{V}<\mathrm{o})$ and $\mathrm{CO}$ oxidation at high potential $(\mathrm{V}>0)$. The usual features are observed (Figure 1c) for the $\mathrm{CO}(\mathrm{C} / 10)$ solution: 1) a small oxidation peak in the range $[-250 ;-50 \mathrm{OMV}$, with a maximum value at -10omV] corresponding to the desorption of adsorbed hydrogen; 2) the onset of $\mathrm{CO}$ oxidation and 3) the hydrogen evolution current increase below $250 \mathrm{mV}$ in the reduction wave. The raise of the CO oxidation current happens at higher potentials than usually observed for CO saturated ${ }^{40,43}$ and alcohol solutions $\mathrm{s}^{30,33}$. The actual values depend on the potential scan rate ${ }^{44}$, electrode preparation procedure ${ }^{43}$, hence platinum surface reconstruction, and solute concentration ${ }^{45}$ with the following trends: for unreconstructed (1xi) surfaces, the CO (resp. alcohol) oxidation peaks at $650-690 \mathrm{mV}^{30,40,43}$ whereas, for (1x2) reconstructed platinum, a pre-oxidation peak is located at $530-550 \mathrm{mV}$ before the main wave around $740 \mathrm{OV}^{33,43}$. In the present case, the cooling procedure in air favors the unreconstructed (1xi) surface ${ }^{40}$. In the present $\mathrm{CO}(\mathrm{C} / 10)$ case, no oxidation signal appears before $650 \mathrm{mV}$, and the main peak of $\mathrm{CO}$ oxidation is shifted above $+1000 \mathrm{mV}$. This is a sign that the kinetics of bulk $\mathrm{CO}$ oxidation is slowed down by the high concentration of $\mathrm{CO}$ molecules inside the solution. Analogous voltammograms are obtained in the thin and thick layer configurations, hinting at the fact that the processes do not depend on diffusion in the solution.

For the CO-saturated solution, the situation is more spectacular. In Figure 1a, we observe no sign of either $\mathrm{CO}$ oxidation or hydrogen evolution in a potential range as wide as $[-1200 ;+1500 \mathrm{mV}]$. The first signs of $\mathrm{CO}$ oxidation appear around $+1600 m V$ (Figure $1 b$, upper curve). After one cycle above this potential, the expected hydrogen desorption (1) and CO oxidation (2) signals appear as small current densities. The $\mathrm{CO}$ oxidation peak is located in the same potential region $(\sim+1000 \mathrm{mV})$ as for the $(\mathrm{C} / 10)$ solution. When cycling up to $[-2000 ;+2000 m V]$, the electrode itself starts to oxidize (3), then during the reduction wave a double peaked current (4) is the sign of the reduction of the platinum oxide. Hydrogen evolution is still shifted to very low potentials (5), a sign that the surface is still blocked by adsorbed species.
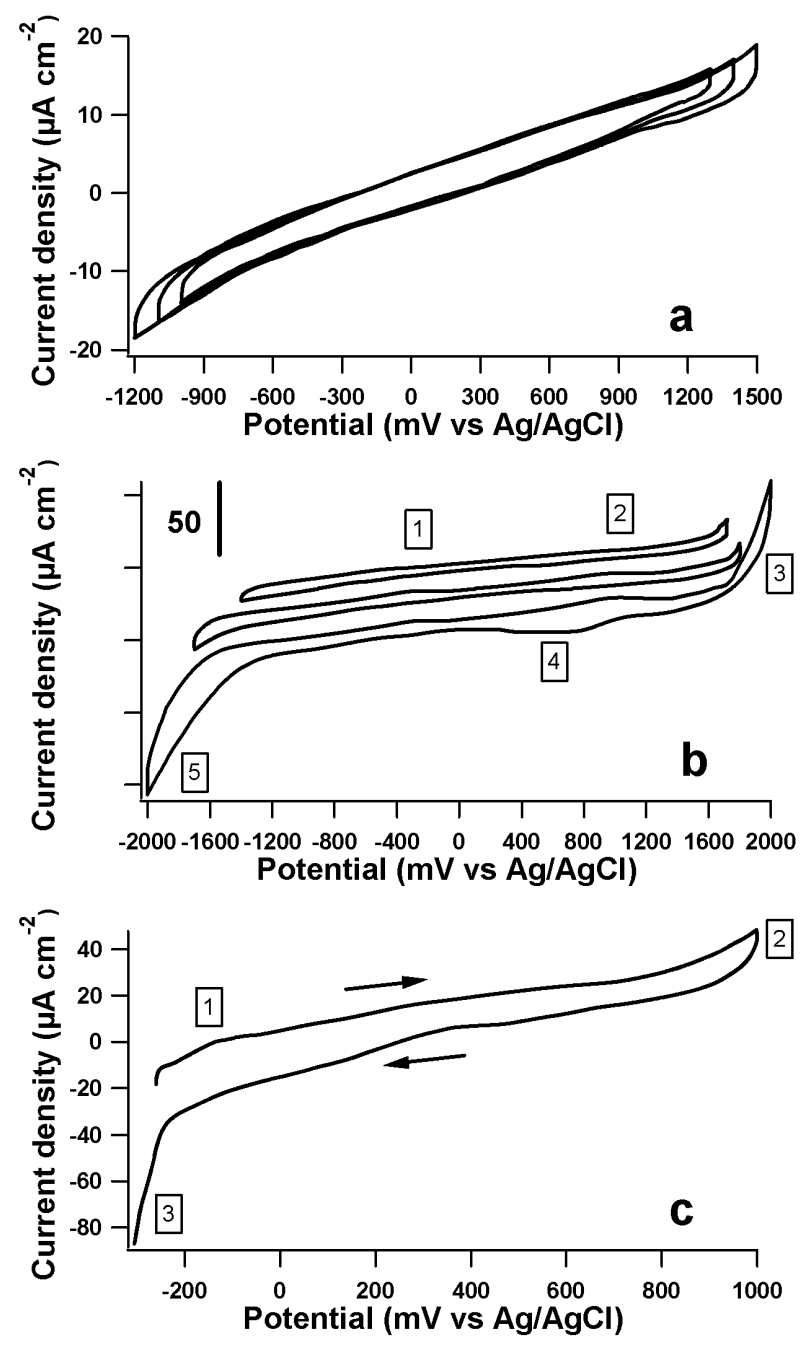

Figure 1. Typical voltammograms of the $\mathrm{Pt}(110)$ electrode in (a,b) CO-saturated and (c) CO (C/10) solutions (resp. 5omVs ${ }^{1}$ and $\left.10 \mathrm{mVs}^{-1}\right)$. In (a) and (b), the potential range has been sequentially extended from $[-1000 ;+1300 m V]$ to $[-$ 2000;+200omV]. The three voltammograms in (b) have been offset for clarity.

At this stage, we favor the interpretation of the cyclic voltammetric curves in the following way: the $\mathrm{CO}$ saturated solution being very concentrated, the layer of adsorbed $\mathrm{CO}$ becomes very dense and compact. In addition, a constant renewal of $\mathrm{CO}$ molecules from the solution allows the monolayer to remain stable even in extreme conditions. For the highest surface density in $\mathrm{CO}$, and according to the Langmuir-Hinshelwood mechanism for $\mathrm{CO}$ oxidation, there are so few possibilities for oxidizing species to co-adsorb on the surface that $\mathrm{CO}$ oxidation does not occur before very high potentials are reached. Some oxidation processes happen anyway on surface defects or at the less packed boundary of $\mathrm{CO}$ islands, but they are of limited extent and efficiently counterbalanced by readsorption. In the same way, the monolayer passivates the platinum surface in the reduction region, thus preventing the normal hydrogen evolution phenomenon to occur. When the concentration is reduced by a factor of 10, the same process happens on the surface upon $\mathrm{CO}$ adsorption. However, $\mathrm{CO}$ starts being 
oxidized slowly above $650 \mathrm{mV}$, probably because molecular renewal is not as fast as in the highest concentration case, and the number of oxidation sites is higher than in the previous case. The presence of the hydrogen evolution at a normal potential suggests that enough adsorbed $\mathrm{CO}$ has been removed from the surface, so that it becomes accessible for the usual chemical processes.

Cyclic voltammetry clearly evidences strong differences between the two solutions, but it provides only a partial view of the processes occurring at the interface as a function of the potential. For slow processes, they may require more time to fully take place than allowed by the potential sweep. This is even more true when working in the thin layer configuration, for which the measured currents are smaller in this case because of the limited electrical path for the charges flowing from the electrode surface to the counter-electrode. In addition, mass transport and diffusion is limited by the small electrolyte thickness. This has been nicely evidenced in a previous study ${ }^{46}$, where average times of around 150 seconds where necessary in order to oxidize the CO layer at the surface of $\mathrm{Pt}(111)$, even at rather high potentials. Such kinetic limits where used to account for the peculiar results obtained in a previous stripping voltammetry study ${ }^{47}$, and confirmed later on by additional data on the shift of the $\mathrm{CO}$ stripping peak towards high potentials as a function of voltammetry scan rates ${ }^{48}$. We note here that the stripping measurements are of a different nature, as they allow the full process to evolve until its completion at each potential step. In order to compare the voltammetry behavior and the steady-state processes, we performed series of SFG spectra on the Pt(110) surface in contact with the two solutions above. As explained in the Experimental section, it takes much more than 15 os to record a SFG spectrum, so each potential step is enough for the complete processes to occur. As a consequence, SFG imposes more constraints on the values for the $\mathrm{CO}$ stability range on the platinum surface.

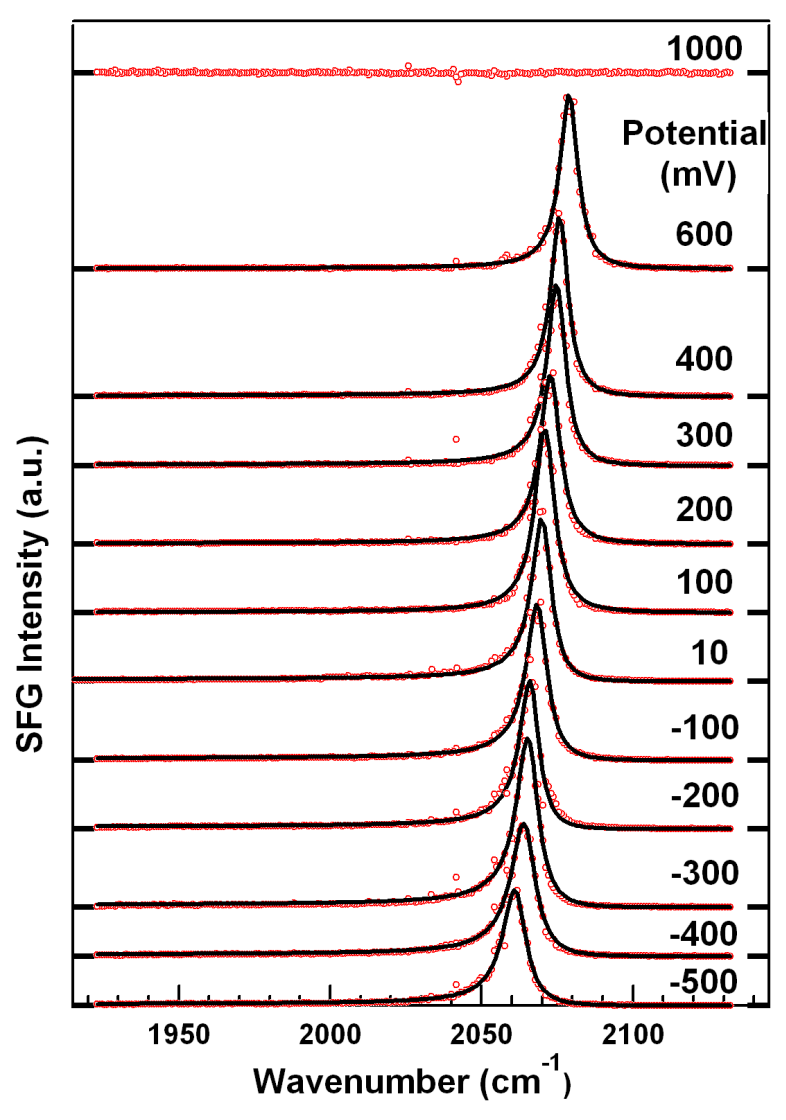

Figure 2. IR-Vis SFG spectra of the CO-saturated layer deposited on $\mathrm{Pt}(110)$ for applied potentials ranging from $500 \mathrm{mV}$ to $1000 \mathrm{mV}$ [vs. $\mathrm{Ag} / \mathrm{AgCl} / \mathrm{KCl}(3 \mathrm{M})$ ]. Lines are fits to the data. Same SFG intensity scale for all spectra.

In Figure 2, we present a series of SFG spectra in the CO stretch region recorded in the same conditions as the voltammograms of Figure 1, for the CO-saturated solution. The dominant spectroscopic feature is the presence of the well-known $\mathrm{CO}$ internal stretching vibration mode located between 2060 and $2080 \mathrm{~cm}^{-1}$ as a marker of the chemical adsorption of $\mathrm{CO}$ molecules on atop site of the $\mathrm{Pt}(110)$ terraces. The peak position undergoes an upward shift with increasing potential as a result of the Stark effect. No sign of a peak due to $\mathrm{CO}$ adsorbed on surface defects can be seen at lower frequency ${ }^{30}$. The $\mathrm{CO}$ peak disappears at $+1000 \mathrm{mV}$, indicating that the $\mathrm{CO}$ layer has been fully oxidized at this potential. This seems to contradict the voltammograms of Figure 1a. However, as already discussed above, the kinetics involved is rather different here. The long exposure time during one SFG spectrum allows the very slow $\mathrm{CO}$ oxidation process to occur completely. This means that the oxidation onset occurs between +600 and $+1000 \mathrm{mV}$. In another series of spectra recorded in the same conditions but for fewer potentials, we have seen that the amplitude of the $\mathrm{CO}$ peak starts decreasing at $+700 \mathrm{mV}$. Nevertheless, using the results of Figure $1 \mathrm{a}$ and $\mathrm{ib}$, the $\mathrm{CO}$ adlayer remains stable up to $+1500 m V$ provided that the exposure is limited to a short time. This could lead to the possibility to perform fast spectroscopic measurements in the $[+700 ;+1500 \mathrm{mV}]$ potential range with a high stability of the $\mathrm{CO}$ layer. No 
features related to the adsorption/desorption of hydrogen are detected either, indicating a full blocking of the electrode's surface at such high CO concentration in the negative potential region. This is rather new indeed, as the published data on spectroscopic studies of this kind of interfaces in acidic electrolyte usually don't proceed below -20omV vs $\mathrm{Ag} / \mathrm{AgCl}$, where the competition between adsorbed $\mathrm{CO}$ and hydrogen evolution favors the latter (the situation is different for basic ${ }^{49}$ or neutral $\mathrm{pH}^{13}$ solutions). Using the same arguments as for the positive potential region, we believe that a stable $\mathrm{CO}$ layer could be studied by fast spectroscopy tools down to -130omV. A very high concentration of $\mathrm{CO}$ solution therefore results in a dramatic quench of the hydrogen evolution and a more stable interface at positive potentials.

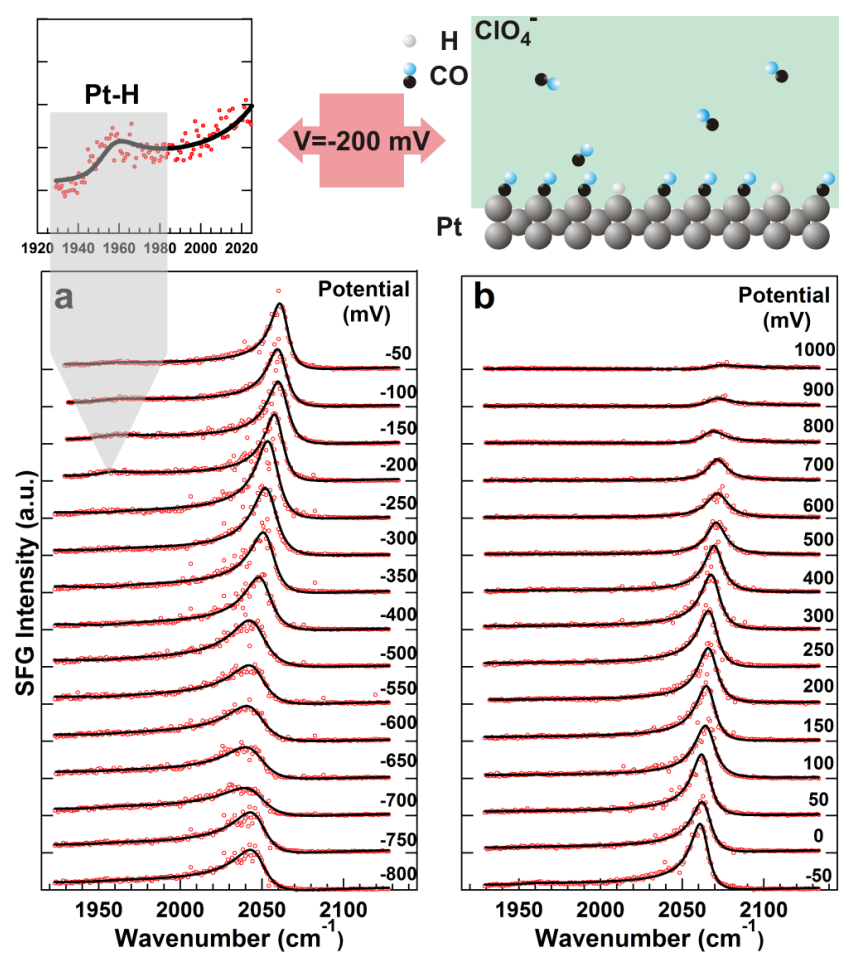

Figure 3. IR-Vis SFG spectra of the CO layer deposited from $\mathrm{CO}(\mathrm{C} / 10)$ solution on $\mathrm{Pt}(110)$ for applied potentials ranging from $-800 \mathrm{mV}$ to $-50 \mathrm{mV}$ (a) and $-50 \mathrm{mV}$ to $1 \mathrm{~V}$ (b) [vs. $\mathrm{Ag} / \mathrm{AgCl} / \mathrm{KCl}(3 \mathrm{M})]$. Lines are fits to the data. Same SFG intensity scale for all spectra. Zoom of the Pt-H mode for $\mathrm{V}=-$ $200 \mathrm{mV}$ with a sketch of the corresponding interface.

Figure 3 displays the analogous series of SFG spectra for the $\mathrm{CO}(\mathrm{C} / 10)$ solution. The same main $\mathrm{CO}$ peak in atop geometry is seen over the whole potential range, even if its amplitude seems to decrease from $+400 \mathrm{mV}$ upwards, and $-500 m V$ downwards. The FWHM of the $\mathrm{CO}$ vibration peak looks bigger than on Figure 2, and increases at the lowest potentials. In this set of data, we observe a small additional peak at $1955 \mathrm{~cm}^{-1}$ around $-200 \mathrm{mV}$, attributed to the Pt-H stretch (as evidenced in other experiments for similar catalysts ${ }^{50}$ ), which correlates to the hydrogen adsorption/desorption peak in the voltammogram of Figure 1c. The SFG cross section of the Pt-H vibration being lower than that of $\mathrm{CO}$ as a consequence of its weak infrared dipole moment, the relative amplitudes of both peaks don't compare directly to their relative content on the surface. However, the amount of adsorbed hydrogen remains low as will be shown below from the quenching of hydrogen evolution as a consequence of the dense $\mathrm{CO}$ coverage. As above, even if the presence of measurable $\mathrm{CO}$ signals up to $+900 \mathrm{mV}$ is not new ${ }^{33}$, the stability of the $\mathrm{CO}$ layer down to $-800 \mathrm{mV}$ is rather unusual in acidic media.

In order to analyze quantitatively the spectra of Figure 2, relevant parameters are extracted after fitting along the equation of the SFG intensity: $:^{25}$

where

and

and stand for the effective nonlinear second order susceptibilities of the interface, platinum electrode and $\mathrm{CO}$ molecules, respectively. These are complex values where the $\mathrm{CO}$ internal stretching vibration mode is described by a lorentzian oscillator of amplitude $\mathrm{a}_{\mathrm{CO}}$, frequency and damping constant .

For the spectra of Figure 2, considering that the natural evolution of the phase shift between the Pt substrate and $\mathrm{CO}$ adsorbate contributions after the fitting process always leads to a number very close to $180^{\circ}$, we have chosen to fix it to this value whatever the applied potential. It is the same as that established for an incomplete monolayer of $\mathrm{CO}$ adsorbed on $\mathrm{Pt}(110)$ in a reactor ${ }^{25}$, and means that the platinum contribution is a negative real number. In order to fit the data set of Figure 3, we take into account the presence of the second vibration mode:

where the supplementary contribution to the SFG signal is related to the presence of the

$\mathrm{Pt}-\mathrm{H}$ stretching vibration mode. From these fits of the SFG data, one deduces that Pt-H is present in the [-250 $\mathrm{mV} ;-50 \mathrm{mV}]$ potential range only, and located at $1955 \mathrm{~cm}^{-}$ ${ }^{1}\left(=15 \mathrm{~cm}^{-1}\right)$.

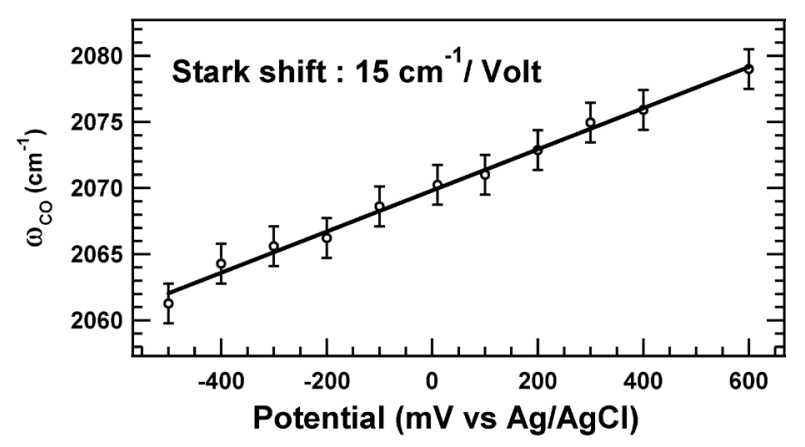

Figure 4. $\mathrm{CO}$ frequency shift at the $\mathrm{CO} / \mathrm{Pt}(110)$ interface for the CO-saturated solution with respect to the applied poten- 
tial. The line is a fit to the data showing the Stark shift slope $\left(15 \mathrm{~cm}^{-1} \mathrm{~V}^{-1}\right)$.

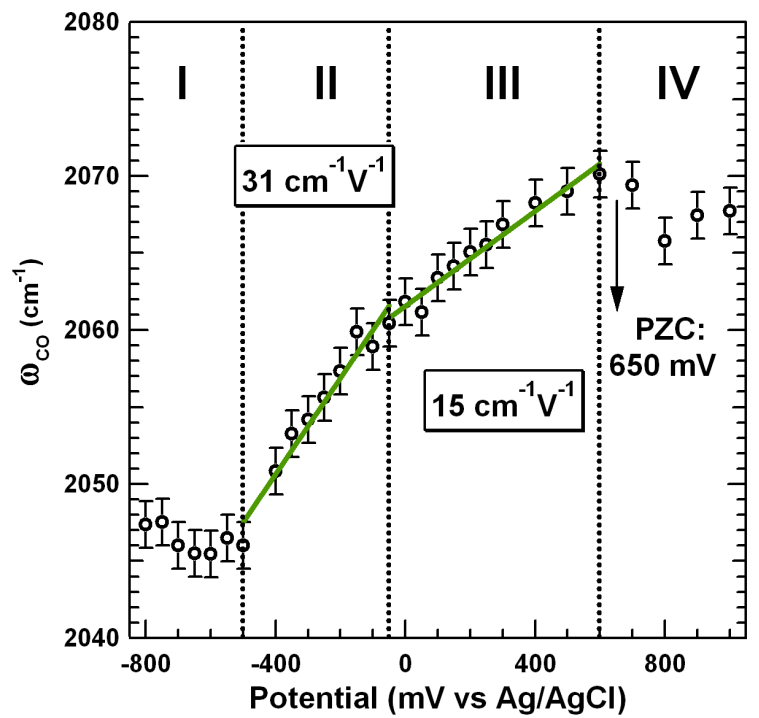

Figure 5. $\mathrm{CO}$ frequency shift at the $\mathrm{CO} / \mathrm{Pt}(110)$ interface for the $\mathrm{CO}(\mathrm{C} / 10)$ solution with respect to the applied potential. The green lines are linear fits for zones II and III.

The resonance frequency, together with its associated Stark shift, is shown for both systems on Figures 4 and 5 . For the CO-saturated case, it remains linear as a function of the potential over more than $1 \mathrm{~V}$, with a slope of $15 \mathrm{~cm}$ ${ }^{1} \mathrm{~V}^{-1}$. For the less saturated solution, the situation is more complex. From the variation of the Stark shift, we identify four potential ranges: I) [-8oo; $-450 \mathrm{mV}]$; II) [-450; $50 \mathrm{mV}]$; III) [-50; +60omV]; IV) [+60o; +100omV]. The green lines are fits to the data showing the two different Stark shift slopes in zones II $\left(31 \mathrm{~cm}^{-1} \mathrm{~V}^{-1}\right)$ and III $\left(15 \mathrm{~cm}^{-1} \mathrm{~V}^{-}\right.$ $\left.{ }^{1}\right)$. No significant Stark shift is observed in zones I and IV where frequencies are set to $2046 \pm 2 \mathrm{~cm}^{-1}$ and $2069 \pm 1 \mathrm{~cm}^{-1}$, respectively. This behavior can be accounted for by the competitive processes: hydrogen evolution in Zone $\mathrm{I}^{35}$ and $\mathrm{CO}$ oxidation in Zone IV. Comparing the values of the $\mathrm{CO}$ vibration frequency shows a $10 \mathrm{~cm}^{-1}$ difference in the positive potential region between the two solutions, although the Stark shift slope is the same. The highest value (COsaturated, $\sim 2070-2080^{-1}$ ) is compatible with the literature for $\operatorname{Pt}(110)$ in aqueous solution ${ }^{38,46}$ for the surfaces which have not experienced hydrogen adsorption. The lower values $\left(\mathrm{CO}(\mathrm{C} / 10), \sim 2060-2070 \mathrm{~cm}^{-1}\right)$ are compatible with a (1X2) reconstruction ${ }^{33}$. However it is very unlikely that such a reconstructed surface may hold with our preparation method. Although we cannot dismiss the possibility of slightly different platinum surface structures (for example as a consequence of hydrogen co-adsorption in the negative potential range) as the cause of frequency shifts, we favor an explanation in terms of dipole-dipole coupling inside the CO layer: a more densely packed layer gives rise to a stronger coupling and therefore induces a shift of the resonance frequency towards higher values ${ }^{35}$. As an illustration, we may evaluate a frequency shift induced by a variation of the average tilt angle of adsorbed $\mathrm{CO}$ molecules. The resonance frequency may be approximated by ${ }^{40}$ : where $\Omega_{\mathrm{CO}}, \alpha_{\mathrm{v}}, \alpha_{\mathrm{e}}$ and $\tilde{\mathrm{U}}$ are the singleton frequency, vibrational polarizability, electronic polarizability and geometric constant describing the dipole-dipole interaction, respectively. The latter depends on the tilt angle (the straighter the molecules, the bigger the coupling). Using for the first three parameters above the following values ${ }^{40}$ : $2016 \mathrm{~cm}^{-1}, 0.31 \AA^{3}, 2.5 \AA^{3}$, and the calculated evolution of $\tilde{U}$ as a function of the tilt angle from the same reference, a change of $15^{\circ}$ for the $\mathrm{CO}$ molecule orientation (e.g. from 16.5 to $31.5^{\circ}$ ) is sufficient to induce a $7 \mathrm{~cm}^{-1}$ decrease in the resonance frequency.

For both interfaces, the Stark shift remains essentially linear (except for zones I and IV of Figure 5). This observed linearity proves that there is no change in the dipole-dipole coupling within potential zones II and III, or for all the potential range in Figure 4. The local structure around a $\mathrm{CO}$ molecule does not change in each zone. The values of the Stark shift slopes require some comment. In zone II, we measure the classical slope for such an interface (around $30 \mathrm{~cm}^{-1} \mathrm{~V}^{-1}$ ) 33,46 , except that it happens in part in a potential range [-400;-200mV] usually inaccessible in an acidic medium, for which hydrogen evolution may occur. The reason for this is given below. The peculiar Stark shift value of $15 \mathrm{~cm}^{-1} \mathrm{~V}^{-1}$ observed on both interfaces for broad potential ranges is in line with the value obtained in the literature when bulky cations are present. ${ }^{38}$ This suggests that the CO adlayer is so densely packed that it prevents ions to access the electrode surface. The outer Helmholtz plan is thus shifted farther from the electrode, spreading the potential drop over a thicker double layer and reducing the effective electric field inside the CO layer. This is consistent with the extended chemical poisoning effect of the dense $\mathrm{CO}$ layer towards positive potentials, which also prevents oxidizing species to co-adsorb and remove the surface CO molecules. When the $\mathrm{CO}$ layer does not cover completely the surface (in zone II for the $\mathrm{CO}(\mathrm{C} / 10)$ solution), the usual behavior is retained and the Stark shift goes back to a conventional value around $30 \mathrm{~cm}^{-1} \mathrm{~V}^{-1}$. This almost perfect blocking property of the $\mathrm{CO}$ layer (even in the hydrogen evolution region for the saturated $\mathrm{CO}$ case) has to be related to the absence of surface defects. It is easily understood that such an unusual behavior would not be possible if $\mathrm{CO}$ could adsorb on defects, leading to local sites of less dense packing where its oxidation would be favoured. ${ }^{30}$ 


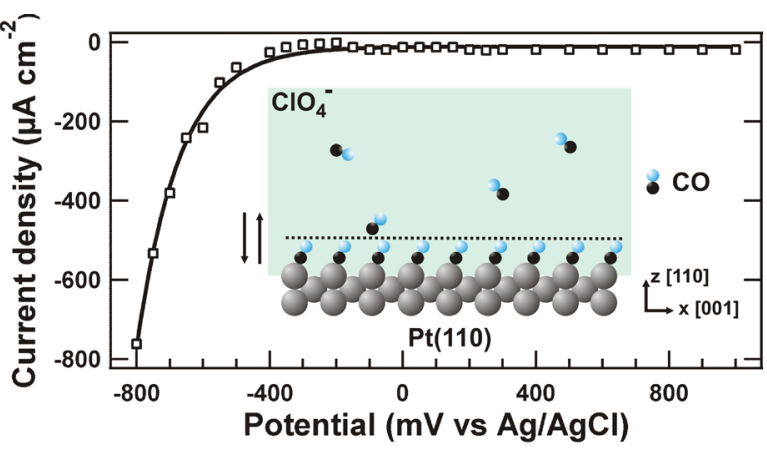

Figure 6. Evolution of the stationary current density at the $\mathrm{CO} / \mathrm{Pt}(110)$ interface for the $\mathrm{CO}(\mathrm{C} / 10)$ solution with respect to the applied potential. The line is a guide to the eye.

One may wonder why the boundaries of the four zones appear at the values shown in Figure 5. In particular, the value around $-500 \mathrm{mV}$ for the first transition is not accounted for. The explanation lies in Figure 6, which displays the steady-state interface current, as recorded in parallel for each SFG spectrum in the $\mathrm{CO}(\mathrm{C} / 10)$ case. Such a current mainly records the events implying bulk moieties rather than surface-only phenomena. Hydrogen evolution is the only clear marker recorded, and it disappears around $-450 \mathrm{mV}$, at the boundary between zones I and II. At this potential, it appears that the presence of $\mathrm{CO}$ quenches the major part of the reduction process, still the potential is too low for the presence of stable adsorbed hydrogen. Above $-250 \mathrm{mV}$, the normal threshold for hydrogen evolution is reached, enabling co-adsorption of hydrogen and $\mathrm{CO}$ at the surface. The mixed layer reorganizes in zone II as hydrogen tends to desorb and $\mathrm{CO}$ packs more and more densely. We stress on the fact that the SFG series has been recorded in a sequence different from the voltammogram (Figure 1c). For the latter, hydrogen evolution appears below $-200 \mathrm{mV}$ because the round trip towards +10oomV has induced partial CO oxidation, leaving accessible spots for $\mathrm{H}_{2}$ evolution. During the SFG spectra, the potential was directly set to negative values then stepped up, leading to a limited hydrogen adsorption.

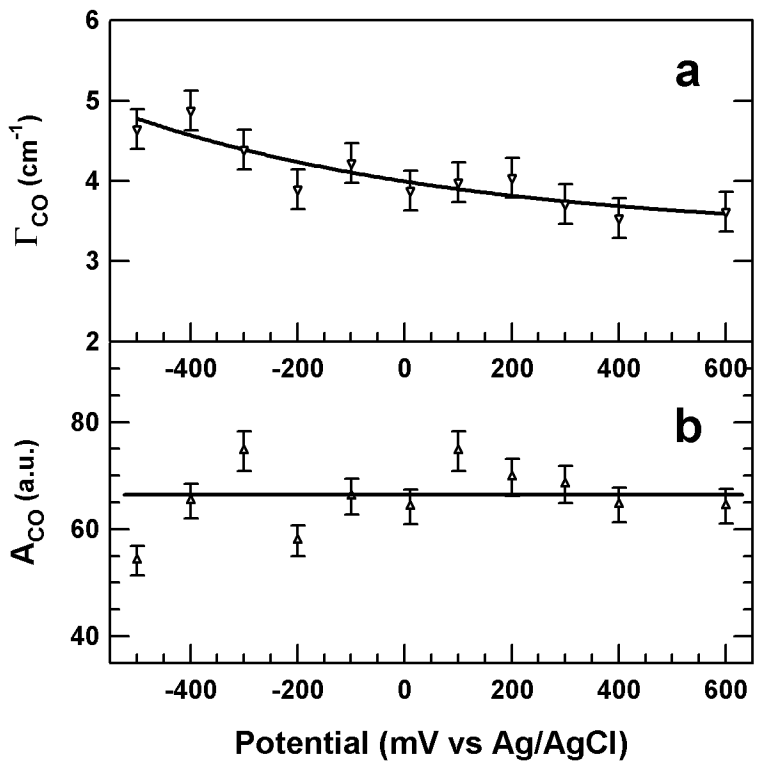

Figure 7. Damping constant (a) and vibration area (b) of the $\mathrm{CO}$ internal stretching vibration mode at the $\mathrm{CO} / \mathrm{Pt}(110)$ interface (CO-saturated layer) with respect to the applied potential. The lines are guides to the eye.

Other parameters relevant for the SFG data analysis are displayed in Figures 7 and 8. Together with Figure 4, Figure 7 illustrates the great stability of the interface for the $\mathrm{CO}$ saturated solution. In Figure $7 \mathrm{a}, \quad$ evolves from 4.9 to $3.6 \mathrm{~cm}^{-1}$. Such a very low value of (we have never experienced such a low value for $\mathrm{CO}$ on platinum with our SFG setup in the past) indicates a high ordering and homogeneity of $\mathrm{CO}$ adsorbed layer due to the high molecular coverage. In addition, increasing the applied potential induces a slow and regular decrease of the linewidth. This phenomenon may have several origins, all related to the compression and additional order inside the $\mathrm{CO}$ adlayer. As the dipole-dipole interaction remains stable with the increase of the potential, it may be due to a low kinetics of CO ordering, to a reduction of the inhomogeneous contribution to or to an increase of the motional narrowing. The width and shape of the resonance are a complex combination of homogeneous components, inhomogeneous contributions, dipole-dipole coupling and additional sources of pure dephasing ${ }^{51}$. At this stage, it is rather difficult to conclude on this point, and we refer the reader to an interesting discussion about the linewidth of $\mathrm{CO}$ on $\mathrm{Pt}$ in a previous study ${ }^{34}$. As stated in this reference, additional dedicated experiments are needed to fully account for the evolution of the linewidths. We point out that the CO-saturated/Pt(110) system investigated here is a very good candidate for a fine analysis of linewidth evolution, as this parameter is mainly the only one changing its value over more than one volt. As an illustration, the $\mathrm{CO}$ vibration area $\left(\mathrm{A}_{\mathrm{CO}}=\right.$

), a marker of the number of $\mathrm{CO}$ molecules present on the surface, does not vary significantly over a wide potential range as observed in Figure $7 \mathrm{~b}$. 


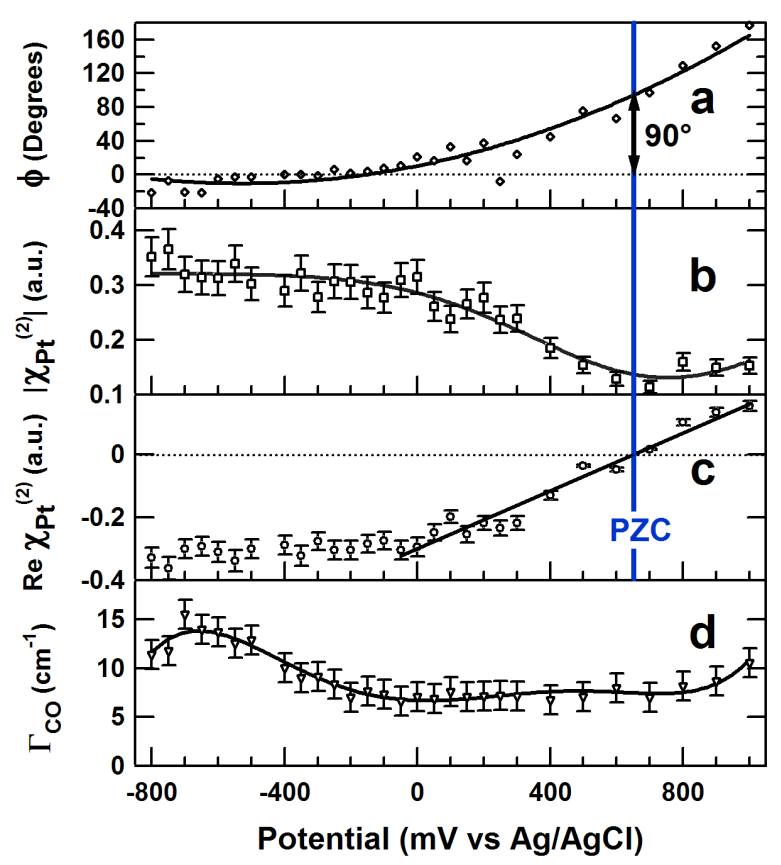

Figure 8. Evolution of the argument (a), modulus (b) and real part (c) of the non-resonant susceptibility of platinum and damping constant $\quad$ (d) of the $\mathrm{CO}$ internal stretching vibration mode at the $\mathrm{CO} / \mathrm{Pt}(110)$ interface $(\mathrm{CO}$ $(\mathrm{C} / 10)$ solution) with respect to the applied potential. The lines in (a),(b),(d) are guides to the eye. A linear fit in (c) defines the $\mathrm{PZC}$ at $\mathrm{V}=650 \mathrm{mV}$ [vs. $\mathrm{Ag} / \mathrm{AgCl} / \mathrm{KCl}(3 \mathrm{M})$ ], see text for details.

It is interesting to note however that the linewidth for the $\mathrm{CO}(\mathrm{C} / 10)$ solution (Figure $8 \mathrm{~d})$ is roughly twice that for the saturated solution, and closer to usual the values measured by SFG on such systems. In zone II, this is likely due to an ordering process as a function of the potential, as decreases continuously over that range, in which there is still a competition between hydrogen and CO. As the hydrogen content decreases, $\mathrm{CO}$ organizes better, accounting for the decreasing linewidth. In zone III, the linewidth remains essentially constant, as well as the dipole coupling, as noted above. However, the $10 \mathrm{~cm}^{-1}$ difference for the resonance frequency and the double value for indicate that the local $\mathrm{CO}$ structures differ from the CO-saturated case. In accordance with the explanation given above for the peculiar value of the Stark shift slope in the same potential regions, we favor the hypothesis of a static (not dynamic) difference, i.e. the geometry of the Pt-CO system must differ. Again, we may put forward a quantitatively different dipole-dipole coupling strength, for example due to a change in the tilt angle, to explain the narrowing. Alternate explanations include a change in the balance between motional narrowing and inhomogeneous broadening.

At the boundary between zones II and III, hydrogen completely disappears, leading to a full blocking of the surface by the adsorbed $\mathrm{CO}$, and a change in the slope of the Stark shift. In order to understand the value of the boundary between zone III and IV, we analyze further the fitting parameters in order to determine the potential of zero charge $(\mathrm{PZC})$. The $\mathrm{PZC}$ is defined as the potential where the surface charge (thus the interfacial electric field) changes sign. We discuss here the potential of zero total charge, and not of zero free charge ${ }^{52}$. It is known that the PZC corresponds to the onset of CO oxidation in aqueous solution, as it favors the co-adsorption of oxidizing species on the interface. ${ }^{38}$

For the experimental determination of the PZC from the SFG data, we follow the procedure established $\mathrm{in}^{32}$, and previously applied to the methanol/Pt(110 $)^{33}$ and cyanide on silver ${ }^{32}$ interfaces. The platinum term in the SFG spectra arises from the optical nonlinear response of the platinum electrons. It is therefore the sum of free electron and interband bound electron contributions. It has been shown $^{32}$ that the former is a real number, whereas the latter is essentially imaginary. We may thus write:

In addition, SHG experiments on silver electrodes (for which the free electron response largely dominates) show that the second harmonic intensity follows a quadratic dependence with a minimum value at the $\mathrm{PZC}^{53,54}$. This is attributed to the fact that, in this potential range, the second order susceptibility mainly originates from a third order term proportional to the static electric field across the interface, in other words to the surface charge or, equivalently, to the difference between the applied potential and the PZC (for which the total charge vanishes) ) $^{55}$ :

The situation is a bit more complicated in SFG because these quantities are not measured directly but must rather be evaluated after spectral fitting. However, the consequence for the platinum contribution at the PZC is threefold: its real part vanishes, its modulus is minimal and its phase $\phi$ equals $\pm 90^{\circ}$. Figure $8 \mathrm{c}$ shows the linearity of the real part of in the range $[-50 \mathrm{mV} ;+1000 \mathrm{mV}]$. From the three criteria above (Figure 8a,b,c), we obtain a $\mathrm{PZC}$ around $+650 \mathrm{mV}$. As a consequence, the $\mathrm{CO}$ peaks in the SFG spectra at +600 and $+700 \mathrm{mV}$ have a symmetric shape. Apart from the method presented here, there are not many ways to estimate the PZC: some are electrochemical $^{52}$, others combine electrochemistry with infrared spectroscopic measurements ${ }^{38,39}$. From the literature, estimates of the $\mathrm{PZC}$ for $\mathrm{CO} / \mathrm{Pt}$ system range from $+450 \mathrm{mV}$ for $\mathrm{Pt}(110)-(1 \times 2)^{33}$ to $+850 \mathrm{mV}$ vs $\mathrm{Ag} / \mathrm{AgCl}$ for $\mathrm{Pt}(110)$ and polycrystalline $\mathrm{Pt}$, and $+1050 \mathrm{mV}$ for $\mathrm{Pt}(111)^{38,39}$. However, the accuracy of the convergence method used therein remains low. SFG appears hence as a valuable in situ technique to measure the PZC of complex electrochemical interfaces. The $+650 \mathrm{mV}$ potential corresponds indeed to the start of the $\mathrm{CO}$ oxidation wave on the voltammogram (in Figure 1c) for this interface. This, together with the clear decrease of the amplitude of the $\mathrm{CO}$ stretch in SFG spectra above +6oomV (Figure 3), confirms that the PZC is the onset of $\mathrm{CO}$ oxidation as triggered by 
the coadsorption of oxidizing species ${ }^{33}$. The linear fit of the real part of (Figure 8c) also validates the potential of $-50 \mathrm{mV}$ as the border between zones II and III, primarily determined from the change in the Stark shift. A similar analysis should be possible in principle also for the CO-saturated solution. It should even be simpler as we have seen that the platinum contribution to the SFG spectra has no imaginary part, i.e. it is a negative real number. With increasing potentials, it should experience a fast sign change towards a positive real number at the PZC, making it rather easy to locate ${ }^{33}$. However, the measured absolute values of the non-resonant contribution for the CO-saturated series are small, leading to a rather large uncertainty on their actual value. This, together with the limited amount of available spectra at high potentials and the complete disappearance of the $\mathrm{CO}$ peak at $+1000 \mathrm{mV}$, has prevented us from measuring the PZC in this case.

Merging all the elements above, we may account for the peculiar behavior of the new kind of $\mathrm{CO}$ on $\mathrm{Pt}(110)$ interface investigated here. For the $\mathrm{CO}(\mathrm{C} / 10)$ solution, adsorbed $\mathrm{CO}$ behaves at first glance in a rather usual way as compared to the literature. In the potential range where adsorbed hydrogen and $\mathrm{CO}$ may co-exist [-250;-50mV], a classical Stark shift of $30 \mathrm{~cm}^{-1} \mathrm{~V}^{-1}$ is measured. The CO layer organizes with increasing potential as hydrogen desorbs from the surface. However, there are still some difference with the expected behavior of such a system. Firstly, hydrogen evolution is suppressed down to $-400 \mathrm{mV}$. This shows that the high CO content in the solution induces a CO compact layer, though open to exchanges with the solution and in particular to some hydrogen coadsorption. The classical image of compact CO islands, at the boundary of which $\mathrm{CO}$ and hydrogen compete for the occupation of the adsorption sites, applies here. They grow with the potential until they occupy the whole surface, in agreement with the decrease of the $\mathrm{CO}$ linewidth. This behavior only holds for interfaces prepared in the $\mathrm{CO}$ stability potential range (before the PZC at $+650 \mathrm{mV}$ ). If the potential is increased above this threshold, the $\mathrm{CO}$ layer starts oxidizing, leaving out free sites and enabling usual hydrogen evolution when scanning the potential back below -250mV (Figure 1). Secondly, in the positive potential range (i.e. after removal of hydrogen and spreading of the $\mathrm{CO}$ islands over the whole surface), a new kind of $\mathrm{CO}$ adlayer is formed, characterized by the peculiar (for a system in acidic aqueous solution with small size anions) Stark shift value of $15 \mathrm{~cm}^{-1} V^{-1}$, and holding at least up to the PZC. This parameter is the one which best characterizes the full blocking of the platinum surface by the CO adlayer, as it evidences an outright electric field screening away from the platinum surface.

The CO-saturated solution case illustrates this phenomenon more obviously. According to the stable value of the Stark shift slope, together with the very small CO linewidth and the constant values of the other parameters, the interface is fully blocked in the $[-500 ;+600 \mathrm{mV}]$ range. Following the cyclic voltammetry data, we believe that this range may easily be extended over a much larger potential range, at least for short times at the minute timescale. In the positive potential range, we suggest that the $\mathrm{CO}$ adlayer, above the PZC, remains in a metastable state: it should thermodynamically oxidize but its compactness prevents the adsorption of the necessary oxidizing species, leading to its stability for very high positive potentials. Of course, any disturbance of the layer leads, in this potential region, to a fast oxidation and a rapid disappearance of the $\mathrm{CO}$ adsorbed molecules. This is what we observe in the series of SFG spectra (Figure 2) in this case. We would like to check this hypothesis in the future in order to find the conditions to maintain the metastable $\mathrm{CO}$ layer above $+1000 \mathrm{mV}$ vs $\mathrm{Ag} / \mathrm{AgCl}$ for longer times. An analogous analysis applies to the negative potential range, where any electrochemical process involving hydrogen is suppressed at least down to $-500 \mathrm{mV}$, and, according to the cyclic voltammetry, much further down in a metastable state. We stress here on the fact that the key point is not to observe an adsorbed CO signal on the platinum surface over a broad potential range ${ }^{33}$, but that the adsorbed CO layer is perfectly stable and almost frozen over the range $[-500 ;+600 \mathrm{mV}]$, at least.

\section{CONCLUSION}

We have shown that it is possible to widen the electrochemical window for a stable $\mathrm{CO}$ adlayer on $\mathrm{Pt}(110)$ in acidic aqueous electrolyte by increasing and decreasing the thresholds of the $\mathrm{CO}$ electro-oxidation and hydrogen evolution potentials, respectively. This was made possible by the dissolution of high amounts of gaseous $\mathrm{CO}$ in a perchloric aqueous solution. The comparison between cyclic voltammetry and steady-state data show that, for transient times in the minute time scale, the $\mathrm{CO}$ layer may remain stable in a $[-1200 ;+1500 m V]$ potential range. In the steady state regime, a "frozen" CO layer, characterized by a linear Stark shift with an unconventional slope of $15 \mathrm{~cm}^{-1} \mathrm{~V}^{-1}$, could be maintained in the $[-500 ;+600 \mathrm{mV}]$. We believe that such a layer could remain metastable in wider potential ranges. Decreasing the $\mathrm{CO}$ concentration by a factor of ten allows to recover most of the usual properties of this widely studied system. Still in this case, hydrogen evolution remains quenched for $200 \mathrm{mV}$ below its usual threshold, and the same frozen layer, characterized by its Stark shift slope, is obtained. A PZC of $+650 \mathrm{mV}$ is measured by a careful analysis of the SFG spectra, confirming that this potential corresponds to the onset of oxidation processes at the surface.

The rather straightforward chemistry involved in the preparation of the $\mathrm{CO}$ saturated electrolyte makes it possible to use it in various environments, provided that it experiences no contact with air. The present results suggest that similar behavior of the $\mathrm{CO} /$ metal interface should transfer to other transition metals, provided that the appropriate methods specific to each metal are used throughout the surface preparation and electrochemical experiments. The stability of the $\mathrm{CO}$ adlayer on metal makes it a good candidate for fundamental studies of a model interface over a wide potential range, in aqueous 
solutions rather than the usual nonaqueous electrolytes, in the "frozen" layer regime. Provided that this regime is sufficiently metastable, this would allow steady state measurements far outside the conventional stability region in aqueous solutions. In addition, using fast acquisition methods (e.g. multiplex SFG spectroscopy ${ }^{56}$ ) would lead to the same result even without the need of metastability. Some applications may cover the disentanglement of the various contributions to the molecular linewidths and their evolutions with the potential ${ }^{57,58}$. It also offers the possibility to investigate the charge transfer between the adsorbed $\mathrm{CO}$ layers and the metal. As evidenced in ultra high vacuum conditions, ${ }^{59}$ two-color SFG spectroscopy is a well-suited analytical probe to evidence charge transfer states. Under electrochemical control, their evolution as a function of the potential still remains to be experimentally observed and modeled.

\section{AUTHOR INFORMATION}

\section{Corresponding Author}

\section{abderrahmane.tadjeddine@u-psud.fr}

\section{$+33169153102$ \\ ACKNOWLEDGMENTS}

The authors acknowledge the ERM financial support of the Université Paris-Sud.

\section{REFERENCES}

(1) Masuda, T.; Sonsudin, F.; Singh, P.R.; Naohara, H.; Uosaki, K. Potential-Dependent Adsorption and Desorption of Perfluorosulfonated Ionomer on a Platinum Electrode Surface Probed by Electrochemical Quartz Crystal Microbalance and Atomic Force Microscopy. J. Phys. Chem. C 2013, 117, 15704-15709.

(2) Okawa, Y.; Masuda, T; Uehara, H.; Matsumura, D; Tamura, K; Nishiata, Y.; Uosaki, K. Origin of the Enhancement of Electrocatalytic Activity and Durability of PtRu Alloy Prepared from a Hetero Bi-Nuclear Pt-Ru Complex for Methanol Oxidation Reactions. RSC Adv. 2013, 3, 15094-15101.

(3) Gomes, J. F.; Oliveira, V. L.; Pratta, P. M. P.; Tremiliosi, G. Reactivity of Alcohols with Three-Carbon Atoms Chain on Pt in Acidic Medium. Electrocatalysis-US 2015, 6, 7-19.

(4) Hu, J.; Tanabe, M.; Sato, J.; Uosaki, K.; Ikeda, K. Effects of Atomic Geometry and Electronic Structure of Platinum Surfaces on Molecular Adsorbates Studied by Gap-Mode SERS. J. Am. Chem. Soc. 2014, 136, 10299-10307.

(5) Ikeda, K.; Sato, J.; Fujimonto, N.; Hayazawa, N.; Kawata, S.; Uosaki, K. Plasmonic Enhancement of Raman Scattering on Non-SERS-Active Platinum Substrates, J. Phys. Chem. C 20o9, 113, 11816-11821.

(6) Noguchi, H.; Okada, T.; Uosaki, K. Molecular Structure at Electrode/Electrolyte Solution Interfaces Related to Electrocatalysis. Faraday Discuss. 2009, 140, 125-137.

(7) See, K. A.; Chapman, K. W.; Zhu, L.; Wiaderek, K. M.; Borkiewicz, O. J.; Barile, C. J.; Chupas, P. J.; Gewirth, A. A. The Interplay of $\mathrm{Al}$ and $\mathrm{Mg}$ Speciation in Advanced $\mathrm{Mg}$ Battery Electrolyte Solutions. J. Am. Chem. Soc. 2016, 138, 328-337.

(8) Han, H. L.; Melaet, G.; Alayoglu, S.; Somorjai, G. A. In Situ Microscopy and Spectroscopy Applied to Surfaces at Work. ChemCatChem 2o15, 7, 3625-3638.
(9) Ren, B.; Li, X. Q.; She, C. X.; Wu, D. Y.; Tian, Z. Q. Surface Raman Spectroscopy as a Versatile Technique to Study Methanol Oxidation on Rough Pt Electrodes. Electrochim. Acta 2ooo, 46, 193-205.

(10) Hendriksen, B. L. M.; Frenken, J. W. M. CO Oxidation on Pt(110): Scanning Tunneling Microscopy Inside a High-Pressure Flow Reactor. Phys. Rev. Lett. 2002, 89, 046101 (1-4).

(11) Park, S.; Wasileski, S. A.; Weaver, M. J. Some Interpretations of Surface Vibrational Spectroscopy Pertinent to Fuel-Cell Electrocatalysis. Electrochim. Acta 2002, 47, 3611-3620.

(12) Yu, Q.; Ye, S. In Situ Study of Oxygen Reduction in Dimethyl Sulfoxide (DMSO) Solution: A Fundamental Study for Development of the Lithium-Oxygen Battery. J. Phys. Chem. C 2015, 119, 12236-12250.

(13) Lotti, D.; Hamm, P.; Kraack, J. P. Surface-Sensitive Spectro-electrochemistry Using Ultrafast 2D ATR IR Spectroscopy. J. Phys. Chem. C 2016, 120, 2883-2892.

(14) Tian, C. S.; Shen, Y.R. Recent Progress on Sum-Frequency Spectroscopy. Surf. Sci. Rep. 2014, 69, 105-131.

(15) Roy, S.; Covert P. A.; FitzGerald, W. R.; Hore, D. K. Biomolecular Structure at Solid-Liquid Interfaces as Revealed by Non-Linear Optical Spectroscopy. Chem. Rev. 2014, 114, 83888415 .

(16) Arnolds, H.; Bonn, M. Ultrafast Surface Vibrational Dynamics. Surf. Sci. Rep. 2010, 65, 45-66.

(17) Liljeblad, J. F. D.; Tyrode, E. Vibrational Sum Frequency Spectroscopy Studies at Solid/Liquid Interfaces: Influence of the Experimental Geometry in the Spectral Shape and Enhancement. J. Phys. Chem. C 2012, 116, 22893-22903.

(18) Wang, H.-F.; Velarde, L.; Gan, W.; Fu, L. Quantitative Sum-Frequency Generation Vibrational Spectroscopy of Molecular Surfaces and Interfaces: Lineshape, Polarization, and Orientation. Ann. Rev. Phys. Chem. 2015, 66, 189-216.

(19) Scheu, R.; Roke, S. Toward Vibrational Dynamics at Liquid-Liquid and Nano-Interfaces: Time-Resolved Sum-Frequency Scattering. J. Phys. Chem. B 2014, 118, 3366-3371.

(20) Nihonyanagi, S.; Yamaguchi, S.; Tahara, T. Counterion Effect on Interfacial Water at Charged Interfaces and Its Relevance to the Hofmeister Series. J. Am. Chem. Soc. 2014, 136, 61556158.

(21) Lis, D.; Backus, E. H. G.; Hunger, J.; Parek, S. H.; Bonn, M. Liquid Flow Along a Solid Surface Reversibly Alters Interfacial Chemistry. Science 2014, 344, 1138-1142.

(22) Ebben, C. J. ; Shrestha, M.; Martinez, I.S.; Corrigan, A.L.; Frossard, A. A.; Song, W. W.; Worton, D. R.; Petaja, T.; Williams, J.; Russell, L. M.; et al. Organic Constituents on the Surfaces of Aerosol Particles from Southern Finland, Amazonia and California Studied by Vibrational Sum-Frequency Generation. J. Phys. Chem. A 2012, 116, 8271-8290.

(23) Rupprechter, G. Sum Frequency Generation and Polarization-Modulation Infrared Reflection Absorption Spectroscopy of Functioning Model Catalysts from Ultrahigh Vacuum to Ambient Pressure. Adv. Catal. 2007, 51, 133-263.

(24) Ouvrard, A.; Wang, J.; Ghalgaoui, A.; Nave, S.; Carrez, S.; Zheng, W.-Q.; Dubost, H.; Bourguignon, B. CO Adsorption on $\operatorname{Pd}(100)$ Revisited by Sum Frequency Generation: Evidence for Two Adsorption Sites in the Compression Stage. J. Phys. Chem. C 2014, 118, 19688-19700.

(25) Humbert, C.; Tadjeddine, A.; Busson, B. Sum-Frequency Generation Vibrational Spectroscopy of an Extramolecular Chemical Bond. J. Phys. Chem. Lett. 2011, 2, 2770-2773.

(26) Kweskin, S. J.; Rioux, R. M.; Komvopoulos, K.; Yang, P.; Somorjai, G. A. Carbon Monoxide Adsorption and Oxidation on Monolayer Films of Cubic Platinum Nanoparticles Investigated by InfraredVisible Sum Frequency Generation Vibrational Spectroscopy. J. Phys. Chem. B 2006, 110, 15920-15925.

(27) Su, X.; Cramer, P.S.; Shen, Y.R.; Somorjai, G.A. Pressure Dependence $\left(10^{-10}-700\right.$ Torr $)$ of the Vibrational Spectra of Ad- 
sorbed CO on Pt(111) Studied by Sum Frequency Generation. Phys. Rev. Lett. 1996, 77, 3858-386o.

(28) Backus, E.H.G; Eichler, A.; Kleyn, A. W.; Bonn M. RealTime Observation of Molecular Motion on a Surface. Science 2005, 310, 1790-1793.

(29) Kutz, R.B.; Braunschweig, B.; Mukherjee, P.; Dlott, D.D.; Wieckowski, A. Study of Ethanol Electrooxidation in Alkaline Electrolytes with Isotope Labels and Sum-Frequency Generation. J. Phys. Chem. Lett. 2011, 2, 2236-2240.

(30) Vidal, F.; Tadjeddine, A.; Humbert, C.; Dreesen, L.; Peremans, A.; Thiry, P. A.; Busson, B. The Influence of Surface Defects in Methanol Dissociative Adsorption and CO Oxidation on Pt(110) Probed by Nonlinear Vibrational SFG Spectroscopy. J. Electroanal. Chem. 2012, 672, 1-6.

(31) Carrier, O.; Backus, E.H.G.; Shahidzadeh, N.; Franz, J.; Wagner, M.; Nagata, Y.; Bonn, M.; Bonn, D. Oppositely Charged Ions at WaterAir and Water-Oil Interfaces: Contrasting the Molecular Picture with Thermodynamics. J. Phys. Chem. Lett. 2016, 7, 825-830.

(32) Tadjeddine, A.; Le Rille, A.; Pluchery, O.; Vidal, F.; Zheng, W.-Q.; Peremans, A. Sum and Difference Frequency at the Electrochemical Interface. Phys. Stat. Sol. (a) 1999, 175, 89-107.

(33) Vidal, F.; Busson, B.; Tadjeddine, A. Probing Electronic and Vibrational Properties at the Electrochemical Interface Using SFG Spectroscopy: Methanol Electro-Oxidation on Pt(110). Chem. Phys. Lett. 2005, 403, 324-328.

(34) Lu, G. Q.; Lagutchev, A.; Dlott, D.; Wieckowski, A. Quantitative vibrational sum-frequency generation spectroscopy of thin layer electrochemistry: CO on a Pt electrode Surf. Sci. 2005 585, 3-16.

(35) Persson, B. N. J.; Ryberg, R. Vibrational interaction between molecules adsorbed on a metal surface: The dipole-dipole interaction. Phys. Rev. B 1981, 24, 6954-6970.

(36) Lambert, D. K. Stark effect of adsorbate vibrations. Solid State Comm. 1984, 51, 297-300.

(37) Lambert, D. K. Vibrational Stark Effect of Adsorbates at Electrochemical Interfaces. Electrochim. Acta 1996, 41, 623-63o.

(38) Jiang, X.; Weaver, M. J. The role of interfacial potential in adsorbate bonding: electrode potential-dependent infrared spectra for saturated CO adlayers on Pt(110) and related electrochemical surfaces in varying solvent environments. Surf. Sci. 1992, 275, 237-252.

(39) Roth, J. D.; Weaver, M. J. Role of the Double-Layer Cation on the Potential-Dependent Stretching Frequencies and Binding Geometries of Carbon Monoxide at Platinum-Nonaqueous Interfaces Langmuir 1992, 8, 1451-1458.

(40) Vidal, F.; Busson, B; Tadjeddine, A.; Peremans, A. Effect of a Static Electric Field on the Vibrational and Electronic Properties of a Compressed CO Adlayer on $\mathrm{Pt}(110)$ in Nonaqueous Electrolyte as Probed by Infrared Reflection-Absorption Spectroscopy and Infrared-Visible Sum-Frequency Generation Spectroscopy. J. Chem. Phys. 2003, 119, 12492-12498.

(41) Humbert, C.; Busson, B.; Six, C.; Gayral, A.; Gruselle, M.; Villain, F.; Tadjeddine, A. Sum-Frequency Generation as a Vibrational and Electronic Probe of the Electrochemical Interface and Thin Films. J. Electroanal. Chem. 20o8, 621, 314-321.

(42) Dalstein, L.; Ben Haddada, M.; Barbillon, G.; Humbert, C.; Tadjeddine, A.; Boujday, S.; Busson, B. Revealing the Interplay between Adsorbed Molecular Layers and Gold Nanoparticles by Linear and Nonlinear Optical Properties. J. Phys. Chem. C 2015, 119, 17146-17155.

(43) Marković, N. M.; Grgur, B. N.; Lucas, C. A.; Ross, P. N. Surface electrochemistry of CO on Pt(110)-(1x2) and Pt(110)-(1x1) surfaces. Surf. Sci. 1997, 384, L805-L814.

(44) Couto, A.; Pérez, M. C.; Rincón, A. Gutiérrez, C. Necessity of CO-Free Pt Sites for the Electrooxidation at Low Potentials of Dissolved CO on Polycrystalline Pt. J. Phys. Chem. 1996, 100, 19538-19544.
(45) Gomes, J. F.; Busson, B.; Tadjeddine, A. SFG Study of the Ethanol in an Acidic Medium-Pt(110) Interface: Effects of the Alcohol Concentration. J. Phys. Chem. B 2006, 110, 5508-5514.

(46) Dederichs, F.; Friedrich, K. A.; Daum, W. Sum-Frequency Vibrational Spectroscopy of CO Adsorption on $\mathrm{Pt}(111)$ and $\mathrm{Pt}(110)$ Electrode Surfaces in Perchloric Acid Solution: Effects of ThinLayer Electrolytes in Spectroelectrochemistry. J. Phys. Chem. B 2000, 104, 6626-6632.

(47) Baldelli, S.; Markovic, N.; Ross, P.; Shen, Y. R.; Somorjai, G. Sum Frequency Generation of CO on (111) and Polycrystalline Platinum Electrode Surfaces: Evidence for SFG Invisible Surface CO. J. Phys. Chem. B 1999, 103, 8920-8925.

(48) Chou, K. C.; Markovic, N. M.; Kim, J.; Ross, P. N.; Somorjai, G.A. An in Situ Time-Dependent Study of CO Oxidation on $\mathrm{Pt}(111)$ in Aqueous Solution by Voltammetry and Sum Frequency Generation. J. Phys. Chem. B 2003, 107, 1840-1844.

(49) Zou, S.; Weaver, M. J. Potential-Dependent MetalAdsorbate Stretching Frequencies for Carbon Monoxide on Transition-Metal Electrodes: Chemical Bonding versus Electrostatic Field Effects. J. Phys. Chem. 1996, 100, 4237-4242.

(50) Tadjeddine, A.; Peremans, A. Vibrational Spectroscopy of the Electrochemical Interface by Visible-Infrared Sum Frequency Generation. Surf. Sci. 1996, 368, 377-383.

(51) Jakob, P.; Persson, B. N. J. Dephasing of localized and delocalized vibrational modes: $\mathrm{CO}$ adsorbed on $\mathrm{Ru}(\mathrm{Ooo1})$. Phys. Rev. B 1997, 56, 10644-10650.

(52) Martínez-Hincapié, R.; Berná, A.; Rodes, A.; Climent, V.; Feliu, J. M. Surface Acid-Base Properties of Anion-Adsorbed Species at $\mathrm{Pt}(111)$ Electrode Surfaces in Contact with $\mathrm{CO}_{2}-$ Containing Perchloric Acid Solutions. J. Phys. Chem. C 2016, DOI: 10.1021/acs.jpcc.6boo589.

(53) Guyot-Sionnest, P.; Tadjeddine, A. Study of $\operatorname{Ag}(111)$ and $\mathrm{Au}(111)$ electrodes by optical secohdrmonic generation. J. Chem. Phys. 1990, 92, 734-738.

(54) Aktsipetrov, O. A.; Melnikov, A. V.; Murzina, T. V.; Nikulin, A. A.; Rubtsov, A. N. DC-electric-field-induced optical second harmonic generation at the smooth metal-electrolyte interface. Surf. Sci. 1995, 336, 225-231.

(55) Yagi, I.; Nakabayashi, S.; Uosaki, K. Excitation Wavelength Dependent Three-Wave Mixing at a CO-Covered Platinum Electrode. J. Phys. Chem. B 1997, 101, 7414-7421.

(56) Braunschweig, B.; Mukherjee, P.; Dlott, D. D.; Wieckowski, A. Real-Time Investigations of Pt(111) Surface Transformations in Sulfuric Acid Solutions J. Am. Chem. Soc. 2010, 132, 14036-14038.

(57) Lane, I. M.; King, D. A.; Arnolds, H. The determination of an inhomogeneous linewidth for a strongly coupled adsorbate system. J. Chem. Phys. 2007, 126, 024707.

(58) Bonn, M.; Hess, C.; Roeterdink, W. M.; Ueba, H.; Wolf, M. Dephasing of vibrationally excited molecules at surfaces: $\mathrm{CO} / \mathrm{Ru}(\mathrm{oo1})$. Chem. Phys. Lett. 2004, 388, 269-273.

(59) Chou, K. C.; Westerberg, S.; Shen, Y.R.; Ross, P. N.; Somorjai, G. A. Probing the Charge-Transfer State of CO on $\mathrm{Pt}(111)$ by Two-Dimensional Infrared-Visible Sum-Frequency Generation Spectroscopy. Phys. Rev. B 2004, 69, 153413. 
Table of content graphics

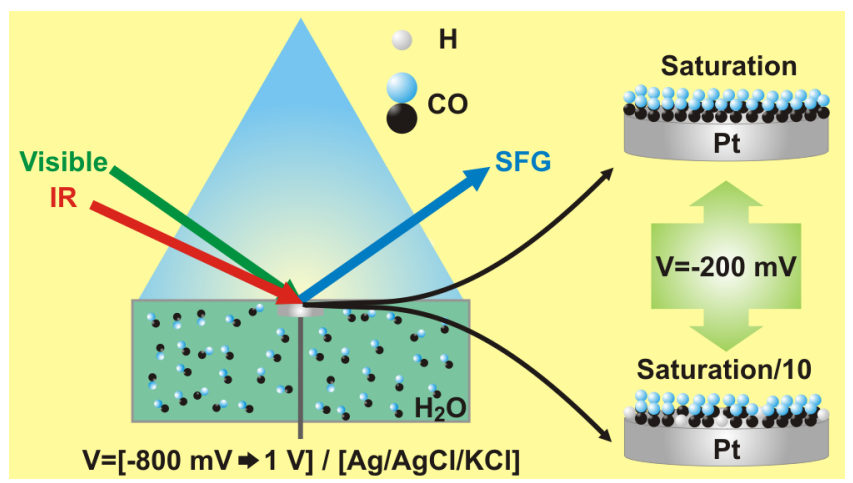

